\title{
Preventing non-attendance in outpatient appointments: predictive model development, validation, and clinical assessment
}

This paper was downloaded from TechRxiv (https://www.techrxiv.org).

\section{LICENSE}

CC BY 4.0

SUBMISSION DATE / POSTED DATE

$30-06-2021 / 13-07-2021$

\section{CITATION}

Valero-Bover, Damià; González, Pedro; Carot-Sans, Gerard; Cano, Isaac; Saura, Pilar; Otermin, Pilar; et al. (2021): Preventing non-attendance in outpatient appointments: predictive model development, validation, and clinical assessment. TechRxiv. Preprint. https://doi.org/10.36227/techrxiv.14885478.v1

$\mathrm{DOI}$ 


\title{
Preventing non-attendance in outpatient appointments: predictive model development, validation, and clinical assessment
}

\author{
Damià Valero-Bover, Pedro González, Gerard Carot-Sans, Isaac Cano, Pilar Saura, Pilar Otermin, \\ Celia Garcia, Maria Gálvez, Francisco Lupiáñez-Villanueva, and Jordi Piera-Jiménez
}

\begin{abstract}
Objective: To develop and validate an algorithm for predicting non-attendance to outpatient appointments. Results: We developed two decision tree models for dermatology and pneumology services (trained with 33,329 and 21,050 appointments, respectively). The prospective validation showed a specificity of $78.34 \%$ (95\% CI 71.07, 84.51) and a balanced accuracy of 70.45\% for dermatology; and 69.83\% (95\%CI 60.61, 78.00) - 65.53\% for pneumology, respectively. When using the algorithm for identifying patients at high risk of non-attendance in the context of a phone-call reminder program, the non-attendance rate decreased 50.61\% ( $\mathrm{P}<.001)$ and 39.33\% ( $\mathrm{P}=.048)$ in the dermatology and pneumology services, respectively. Conclusions: A machine learning model can effectively identify patients at high risk of non-attendance based on information stored in electronic medical records. The use of this model to prioritize phone call reminders to patients at high risk of non-attendance significantly reduced the non-attendance rate.
\end{abstract}

Non-attendance; machine learning; clinical decision making; decision trees

Our algorithm for identifying patients at high risk of nonattendance can be used for prioritizing interventions for preventing non-attendance to outpatient visits.

\section{INTRODUCTION}

$\mathrm{N}^{\circ}$ ON-ATTENDANCE, defined as a missed appointment without prior notification, is an important obstacle for adequate management of healthcare centers. High nonattendance rates are associated with increased waiting lists and healthcare and societal costs, as well as reduced

First submitted: June 30, 2021. The study did not receive specific funding.

D.V-B. is with the Catalan Health Service, and the Digitalization for the Sustainability of the Healthcare System (DS3) group, Barcelona, Spain (email: damia.valero@catsalut.cat).

P.G. is with the Open Evidence Research Group, Universitat Oberta de Catalunya, Barcelona, Spain, and the Universitat Politècnica de Catalunya, Barcelona, Spain. (e-mail: pedrojavi.gonzalez@gmail.com).

G.C-S. is with the Catalan Health Service, and the Digitalization for the Sustainability of the Healthcare System (DS3) group, Barcelona, Spain (email: gerard.carot@ catsalut.cat).

I. C. is with the Hospital Clinic de Barcelona, Institut d'Investigacions Biomèdiques August Pi i Sunyer (IDIBAPS), and University of Barcelona (UB), Barcelona, Spain (e-mail: isaaccf@gmail.com).

P.S. is with the Catalan Ministry of Health, Barcelona, Spain (e-mail: pilarsauraagel@gmail.com). effectiveness and efficiency of the healthcare system [1], [2]. At the patient level, missed appointments may lead to inadequate follow-up and late diagnosis or complication management, thus increasing the health risk of nonattendees. Reported non-attendance rates worldwide are highly heterogeneous and range from $13.2 \%$ (average countries in Oceania) to $43.0 \%$ (Africa); the estimated average rate in Europe is $19.3 \%$ [3].

Various authors have proposed interventions to reduce the harmful effects of non-attendance, such as overbooking [4] and open access [5], or to improve attendance rates directly, for example, by providing information, reminders, and incentives to patients [6]-[8]. Of them, the use of

P.O. is with Badalona Serveis Assistencials, Badalona, Spain (e-mail: potermin@bsa.cat).

C.G. is with Badalona Serveis Assistencials, Badalona, Spain (e-mail: celiagarcia@bsa.cat).

M.G. is with Badalona Serveis Assistencials, Badalona, Spain (e-mail: mgrico@bsa.cat).

F.L-V. is with the Department of Information and Communication Sciences, Universitat Oberta de Catalunya, and Open Evidence Research Group, Universitat Oberta de Catalunya, Barcelona, Spain (e-mail: flupianez@uoc.edu).

J.P-J. is with Catalan Health Service, the Digitalization for the Sustainability of the Healthcare System (DS3) group, and Open Evidence Research Group, Universitat Oberta de Catalunya, Barcelona, Spain (e-mail: jpiera@catsalut.cat). 
appointment reminders based on short message services (SMS) and telephone calls have been widely used [9]-[11]. Although current evidence suggests equal effectiveness of both interventions, reported results are heterogeneous, and most studies have low-quality design [10].

Regardless of the reminding strategy, identifying patients at higher risk of non-attendance may reduce costs and resources, thus increasing the sustainability of the intervention. The determinants of non-attendance are complex and may include patient-related factors (e.g., age and gender), their previous attendance history, and factors associated with the given appointment (e.g., lapse from schedule date, and weekday and season of the appointment) [12], [13]. In the last few years, a growing number of models for predicting no-shows have been proposed; however, most of them achieved an accuracy lower than the attendance rate [14]. The poor performance may be attributed to multiple factors that challenge model development, such as the type of data available or the sample size. Furthermore, the high variability of non-attendance rates worldwide suggests that behavioral determinants of non-attendance and the effectiveness of mitigating measures may depend on the country and healthcare system organization. Therefore, we aimed to develop a model for predicting patients' nonattendance and assess the effectiveness of selective phone calls to patients at high risk of non-attendance according to the resulting model.

\section{A. Predictive model}

\section{1) Variable analysis}

Non-attendance algorithms were developed using data from 33,329 appointments scheduled in the dermatology service and 21,050 in the pneumology service. The global nonattendance rates of these appointments were $20.90 \%$ and $18.37 \%$ for dermatology and pneumology outpatient services, respectively. When comparing the sociodemographic characteristics, appointment characteristics and attendance history of patients who attended the appointment in the dermatology outpatient service and those who not, significant differences were observed in all variables except gender (non-significant) and marital status (significant marginal association) (Table S1, Supplementary material). Similarly, all variables showed a significant association with non-attendance in appointments in the pneumology outpatient service, except gender, physician, and number of reschedules (Table S2). We found no strong correlations between variables, neither categorical nor numerical (Table S3).

\section{2) Selection of the training algorithm}

Table I shows the specificity, sensitivity, and accuracy of the different training algorithms. The algorithm kNN yielded unacceptable results in terms of sensitivity, whereas XGBoost and SVM resulted in similar metric performance values to those of decision trees. Based on the performance of each training algorithm, and also considering the easiness of explanation, the decision trees algorithm was selected.
TABLE I

PERFORMANCE OF TRAINING ALGORITHMS (\%)

\begin{tabular}{|c|c|c|c|c|}
\hline & $\begin{array}{c}\text { DECISION } \\
\text { TREES }\end{array}$ & XGBoost & SVM & $\mathrm{kNN}$ \\
\hline \multicolumn{5}{|l|}{ Dermatology } \\
\hline Specificity & 73.01 & 74.37 & 64.86 & 81.17 \\
\hline Accuracy & 64.78 & 63.80 & 62.62 & 54.91 \\
\hline Sensitivity & 56.56 & 53.22 & 60.63 & 19.38 \\
\hline \multicolumn{5}{|l|}{ Pneumology } \\
\hline Specificity & 58.56 & 54.43 & 55.61 & 94.44 \\
\hline Accuracy & 59.70 & 58.23 & 59.90 & 51.91 \\
\hline Sensitivity & 60.85 & 62.03 & 64.20 & 9.38 \\
\hline
\end{tabular}

$\mathrm{kNN}=\mathrm{k}$-nearest neighbor, $\mathrm{SVM}=$ support vector machines

Decision trees were trained using recursive partitioning within the R library rpart [15]. This library presents an implementation of Classification and Regression Trees (CART), described by Breiman L. et al [16]. The goodness of fit of each split was measured using the Gini impurity. The importance of each variable was calculated as the sum of the goodness of split measures for each split for which it was the primary variable, plus adjusted goodness agreement for all splits in which it was a surrogate. According to the 5-fold cross-validation, the values that optimized the trade-off between specificity, accuracy, and sensitivity of the models were a complexity factor of 0.01 and a minimum depth of the decision tree algorithm of 10 . A loss matrix was used to grow the trees, penalizing type II errors (i.e., false negatives) with a relative weight of 1.2. The relative weight of type I error was set to 1 .

\section{3) Model and prediction performance}

Fig. 1A and Fig. 2A show the design of the resulting predictive models for dermatology and pneumology outpatient services, respectively. In the dermatology predictive model, the patient's history of previous attendance was the most relevant factor to predict non-attendance in the future, followed by major ambulatory surgery, the status of the last appointment, number of prior visits, and age (Fig. 1B). This model displayed a specificity of $79.90 \%$, a sensitivity of $67.09 \%$, and an accuracy of $73.49 \%$. Similarly, in the pneumology predictive model, the patient's previous attendance history was also the most important variable to predict non-attendance, followed by lead time, the status of the last appointment, number of prior visits, and number of days since the last visit (Fig. 2B). The specificity, sensitivity, and accuracy of this model were $71.38 \%, 57.84 \%$, and $64.61 \%$, respectively.

\section{B. Model validation}

The prospective validation of the non-attendance predictive models included 758 and 637 appointments in the services of dermatology and pneumology, respectively. In the dermatology service, the predictive model identified 348 (45.91\%) appointments at high risk (i.e., $\geq 50 \%$ likelihood) of non-attendance, 123 of which were actually missed 


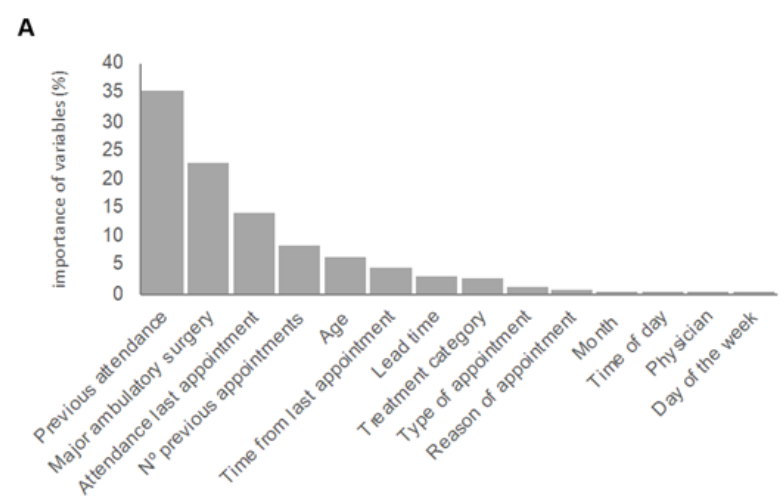

B

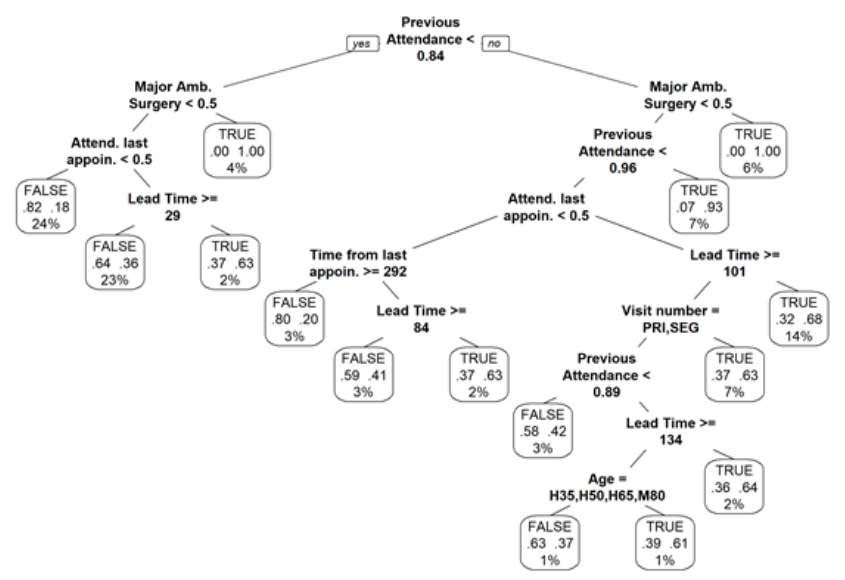

Fig. 1. Dermatology model. A) Relative importance of variables, according to the Gini index. B) Decision tree representation; each leaf includes the following information: probability of the model (true: > 0.5 ; false: $<0.5$ ), probability of each class within the node (values between 0 and 1), and percentage of observations of the node.

appointments. The total number of real non-attendances was 157 , thus yielding a specificity of the model of $78.34 \%$ (95\% CI 71.07, 84.51). The sensitivity and balanced accuracy of this model were $62.56 \%(95 \%$ CI $71.07,84.51)$ and $70.45 \%$, respectively. Correspondingly, 283 (44.43\%) appointments scheduled in the pneumology service were identified as high risk of non-attendance, 81 of which were missed appointments. The total amount of real nonattendances was 116, resulting in a specificity of $69.83 \%$ (95\% CI 60.61, 78.00). The sensitivity and balanced accuracy of the pneumology model were $61.23 \%$ (95\% CI 56.89, 65.43 ) and $65.53 \%$, respectively. Compared with the retrospective validation used during model development, specificity in the prospective validation was reduced by approximately $2 \%$.

\section{Pilot study}

During the study period, 1,311 individuals had at least one appointment to either the dermatology or pneumology outpatient services that was identified as high risk nonattendance according to the selected model. Among them, 1,108 (805 and 303 in the dermatology and pneumology
IEEE OJEMB - SCIENCE
A

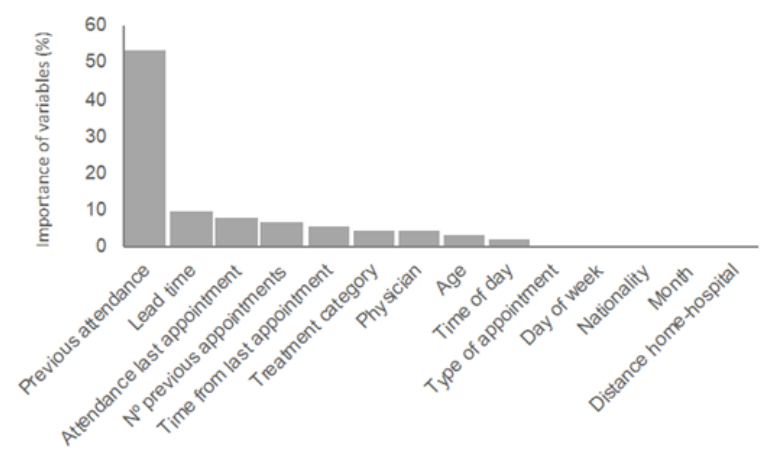

B

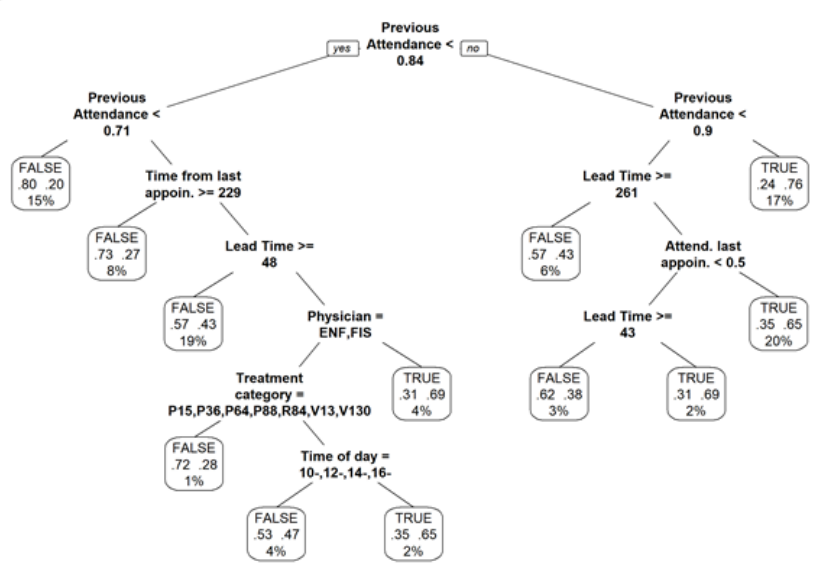

Fig. 2. Pneumology model. A) Relative importance of variables, according to the Gini index. B) Decision tree representation; each leaf includes the following information: probability of the model (true: $>0.5$; false: $<0.5$ ), probability of each class within the node (values between 0 and 1), and percentage of observations of the node.

services, respectively) had available data and were, therefore, included in the analysis. Of the 805 patients with scheduled visits in the dermatology service, 390 (48.45\%) were allocated to the intervention group and $415(51.55 \%)$ to the control group. Correspondingly, 303 individuals had scheduled visits to the pneumology service, 146 (48.18\%) and $157(51.82 \%)$ allocated in the intervention and control groups, respectively. Table II summarizes the baseline characteristics of the individuals enrolled in the pilot study. None of the variables showed significant differences between control and intervention groups, except the time from the last visit among individuals visited at the pneumology service, which was higher in the intervention group than in the control group.

In the dermatology setting, 267 (68.46\%) individuals allocated in the intervention arm were successfully contacted by phone. From which, 251 attended the appointment, and 16 missed it (non-attendance rate 5.99\%). Regarding the pneumology service, $95(65.07 \%)$ individuals of the intervention group were successfully contacted; 86 of them attended the appointment, and 9 did not (non-attendance rate $9.47 \%$ ). The non-attendance rate of each group in each 
TABLE II

BASELINE CHARACTERISTICS OF THE PILOT STUDY POPULATION

\begin{tabular}{|c|c|c|c|c|c|c|}
\hline & \multicolumn{3}{|c|}{$\begin{array}{l}\text { DERMATOLOGY } \\
\quad(N=805)\end{array}$} & \multicolumn{3}{|c|}{$\begin{array}{l}\text { PNEUMOLOGY } \\
\quad(N=303)\end{array}$} \\
\hline & Control & Intervention & $P$ & Control & Intervention & $P$ \\
\hline \multicolumn{7}{|c|}{ Sociodemographic characteristics } \\
\hline \multicolumn{7}{|c|}{ Age group (years), $n(\%)$} \\
\hline $0-14$ & $24(5.78)$ & $28(7.18)$ & & $1(0.64)$ & $0(0.00)$ & \\
\hline $14-18$ & $24(5.78)$ & $24(6.15)$ & & $2(1.27)$ & $0(0.00)$ & \\
\hline $18-25$ & $31(7.47)$ & $31(7.95)$ & & $6(3.82)$ & $0(0.00)$ & \\
\hline $25-35$ & $47(11.33)$ & $36(9.23)$ & & $9(5.73)$ & $3(2.05)$ & \\
\hline $35-50$ & $93(22.41)$ & $89(22.82)$ & 0.971 & $20(12.74)$ & $24(16.44)$ & 0.071 \\
\hline $50-65$ & $74(17.83)$ & $67(17.18)$ & & $54(34.39)$ & $47(32.19)$ & \\
\hline $65-80$ & $81(19.52)$ & $79(20.26)$ & & $54(34.39)$ & $62(42.47)$ & \\
\hline$>80$ & $41(9.88)$ & $36(9.23)$ & & $11(7.01)$ & $10(6.85)$ & \\
\hline Gender (male), $n(\%)$ & $177(42.65)$ & $169(43.33)$ & 0.901 & $92(58.60)$ & $94(64.38)$ & 0.360 \\
\hline \multicolumn{7}{|l|}{ Attendance history } \\
\hline $\begin{array}{l}\text { Attended to the last visit, } n \\
(\%)\end{array}$ & $281(70.96)$ & $284(75.94)$ & 0.340 & $120(76.92)$ & $116(80.56)$ & 0.355 \\
\hline $\begin{array}{l}\text { Major ambulatory surgery, } \\
\text { mean }(S D)\end{array}$ & $0.16(0.36)$ & $0.16(0.37)$ & 0.773 & - & - & - \\
\hline $\begin{array}{l}\text { Rate of attendance of } \\
\text { previous appointments, } \\
\text { mean }(S D)\end{array}$ & $0.75(0.19)$ & $0.76(0.17)$ & 0.853 & $0.77(0.11)$ & $0.78(0.10)$ & 0.499 \\
\hline $\begin{array}{l}\text { Lead time }(\text { days })^{\mathrm{a}}, \text { mean } \\
(S D)\end{array}$ & $125.35(102.1)$ & $123.68(98.47)$ & 0.814 & $188.03(165.64)$ & $216.45(170.64)$ & 0.142 \\
\hline $\begin{array}{l}\mathrm{N}^{\mathrm{o}} \text { of previous visits, mean } \\
(S D)\end{array}$ & $42.96(45.76)$ & $44.37(48.78)$ & 0.681 & $69.93(62.27)$ & 70.33 (60.89) & 0.955 \\
\hline $\begin{array}{l}\text { Time from last visit (days), } \\
\text { mean (SD) }\end{array}$ & $141.53(312.56)$ & $169.11(407.8)$ & 0.291 & $67.32(73.37)$ & $128.95(338.93)$ & 0.028 \\
\hline
\end{tabular}

${ }^{\mathrm{a}}$ Days of waiting since scheduling until the appointment date

TABLE III

NO-SHOWS IN THE PILOT STUDY, NO. $(\%)$

\begin{tabular}{|c|c|c|c|c|}
\hline & $\begin{array}{l}\text { Dermatology } \\
\quad(\mathrm{N}=805)\end{array}$ & $P$ & $\begin{array}{l}\text { Pneumology } \\
(\mathrm{N}=303)\end{array}$ & $P$ \\
\hline Control group & $112(26.99)$ & & $39(24.84)$ & 0.048 \\
\hline $\begin{array}{l}\text { Intervention } \\
\text { group }^{\mathrm{a}}\end{array}$ & $52(13.33)$ & $<0.001$ & $22(15.07)$ & \\
\hline $\begin{array}{l}\text { Not reached } \\
\text { Contacted }\end{array}$ & $\begin{array}{c}36(29.27) \\
16(5.99)\end{array}$ & $<0.001$ & $\begin{array}{c}13(25.49) \\
9(9.47)\end{array}$ & 0.019 \\
\hline
\end{tabular}

${ }^{a}$ Received a reminder telephone call one week before the date of the appointment

clinical setting are given in Table III. Overall, the interventions applied resulted in a significant decrease of the non-attendance rate for both dermatology and pneumology services, with a reduction of non-attendance of $50.61 \%$ and $39.33 \%$, respectively. In both services, non-attendance rates were significantly lower among individuals in the intervention group that were successfully contacted than those who could not be reached $(79.54 \%$ and $62.85 \%$ reductions for dermatology and pneumology services, respectively).

\section{DISCUSSION}

In this study, we found that the models that better predicted non-attendance in dermatology and pneumology outpatient services were based on decision trees and included the following variables: patient's history of previous attendance, major ambulatory surgery, status of the last appointment, number of previous visits, and age, for dermatology, and patient's history of previous attendance, lead time, status of the last appointment, number of previous visits, and number of days since the last visit, for pneumology. The use of the prediction models to identify individuals at high risk of non-attendance for further selective phone call reminders allowed reducing in approximately $50 \%$ and $40 \%$ the non-attendance rate in dermatology and pneumology services, respectively.

The systematic review conducted by Carreras et al. showed that at least half of the studies on no-show prediction identified age, gender, distance from home to the healthcare center, weekday, visit time, lead time, and history of previous attendance as predictors of non-attendance; marital status and visit type (first or successive) were also frequently used [14]. Our findings were mostly in line with the results reported by Carreras et al., although we did not find an association between gender and non-attendance, as reported elsewhere [17], [18]. Other studies described that nonattendance was associated with the number of previous appointments [19], [20], the status of the last appointment [21], [22], and the treatment category (e.g., surgery) [23], which was also consistent with our results. Regarding the relative importance of each variable in the model, the status of the last appointment, age, time of the day, lead time, and history of previous attendance are among the most important variables in the non-attendance predictive models presented 
in various analyses [12], [21], [24]. In our study, the history of previous attendance and the status of the last appointment also had a high weight in both models. In contrast, lead time and age were mainly important in pneumology and dermatology models, respectively. The time of the day had a small weight in both models.

Based on the performance results of the training algorithms, we chose decision trees to build our models, which was the second most frequently used algorithm to develop predictive models in the review of Carreras et al., after logistic regression [14]. The accuracy values reported in the review for models based on decision trees ranged from $76.5 \%$ to $89.6 \%$, higher than the accuracy found in our analysis. However, most studies had a limited sample size and/or used the same dataset for training algorithms and assessing their performance. Alternatively to this approach, which may lead to overfitting, we used an independent dataset for model validation. Therefore, although lower than reported elsewhere, we think our results may better reflect the expected accuracy of the model when applied to the realworld.

Regardless of the validation approach, most studies reported accuracy values lower than the attendance rate [14]. This trend, also observed in our analysis, may be explained by the lack of data from other domains such as social, cultural, and socioeconomic factors that might have a relevant contribution to non-attendance behavior. Finally, we observed a poorer performance of the pneumology model compared with the dermatology model, which might also be due to differences in outpatient procedures and patient complexity between services. These findings suggest that service-specific characteristics and predictors from other domains should be included in the development of prediction models for non-attendance.

Like in our pilot study, other authors have reported nonattendance reductions after implementing reminding strategies based on phone calls [25] or, most frequently, short message services (SMS) [9]-[11]. However, phone calls are more expensive than SMS [9], [26], and both interventions have high costs for healthcare centers. Irrespective of the type of reminder, predictive algorithms may help to prioritize patients at higher risk of non-attendance, which is likely to improve the cost-effectiveness of the intervention. Furthermore, the quantitative approach to the prediction of non-attendance allows combining more or less compelling interventions based on different thresholds of non-attendance risk (e.g., SMS at risk between 50\%-90\%, and phone calls at risk $\geq 90 \%$ ).

The interpretation of our results is limited by the simultaneous assessment of the predictive model and the intervention itself (i.e., phone call reminder), which precluded appraising the contribution of each feature to the non-attendance reduction. However, the main purpose of our pilot study was to assess the applicability of the whole concept to day-to-day practice.
Another limitation was the unavailability of data with potential influence on the non-attendance rate, such as the economic status [27], [28], education level [29], [30], or certain medical conditions [19], [31]. As discussed previously, the lack of social information is common in the development of predictive algorithms elsewhere. Regardless of the future inclusion of these data, the model should undergo continual learning by retraining to assure its validity through time. The model has to be aware of new patients or categorical features, as well as considering up-to-date data to include the latest trends of non-attendance in each hospital service.

\section{CONCLUSIONS}

The results of our study show that the use of nonattendance predictive models can be a valuable tool to identify patients at higher risk of non-attending a medical appointment and should be, therefore, prioritized for active reminders such as phone calls. In our experience, the effectiveness of the intervention disrupted the hospital agenda, which is usually set assuming a certain percentage of non-attendant patients and became overbooked because of our intervention. Although implementation research was out the scope of our study, this experience highlights the need of considering organizational changes when implementing this type of interventions. As future work, we plan to confirm these results using larger administrative databases and assess the cost-effectiveness of the intervention at different nonattendance risk cutoffs.

\section{MATERIALS AND METHODS}

\section{A. Overview of study design}

This study was conducted at two outpatient services (i.e., dermatology and pneumology) of the Hospital Municipal de Badalona (Spain) and included three phases: (1) the development of a non-attendance predictive model for each outpatient service, (2) the prospective validation of the resulting models, and (3) a pilot study to assess the effectiveness of integrating the predictive model into the organization of the healthcare provider.

Candidate models were developed using retrospective data from appointments scheduled between January 1, 2015, and November 30, 2018. Data were randomly assigned to one of the following two sets: $75 \%$ of the collected data were used for model building and algorithm training, and the remaining $25 \%$ were used in a retrospective validation of the model. The predictive capacity of the selected model was then validated prospectively using data from appointments scheduled between January 7 and February 8, 2019. Finally, we conducted a pilot study to assess the effectiveness of a preventive intervention based on selective phone call reminders to patients identified as high-risk of nonattendance according to the selected model. The pilot study was conducted between February 25 and April 19, 2019. 
All data, including retrospective information for model building and prospective information of the pilot study, were collected anonymously and handled according to the General Data Protection Regulation 2016/679 on data protection and privacy for all individuals within the European Union and the local regulatory framework regarding data protection. The pilot study included in this report was not intended to change biomedical or health-related outcomes; therefore, the research committee of Badalona Serveis Assistencials waived the need for ethics committee approval.

\section{B. Variables collected for model development and validation}

We collected three types of variables from the Electronic Medical Record database: sociodemographic characteristics of patients, characteristics of the appointment, and history of patients' attendance. Sociodemographic characteristics included gender, age, nationality, marital status, and home address, which was used to calculate the distance from the patient's home to the hospital. Characteristics of the appointment included hour, weekday, month, type of visit (first, second, successive), the reason for the visit, treatment category, physician, lead time (days since scheduling until the appointment date), and rescheduling. Variables regarding the record of patient's attendance included the history of previous attendance, number of prior visits, days since the last appointment, and the last appointment status.

\section{Predictive model development and validation}

All variables with a significant association with nonattendance were included in training algorithms based on the following models: decision trees, XGBoost, Support Vector Machines (SVM), and k-nearest neighbor (kNN). A 5-fold cross-validation was used in the training, and the class imbalance (approximately, 80\% of attendees and 20\% of non-attendees) was addressed by stratified random sampling. We selected for the model the combination of variables with a better capacity for predicting non-attendance.

The performance of the obtained model was retrospectively assessed using the dataset reserved to this end. Because the model was intended to identify patients at high risk of non-attendance, specificity, defined as the proportion of real non-attendees among all identified by the algorithm as high-risk (i.e., $\geq 50 \%$ likelihood of nonattendance), was used for measuring performance. Sensitivity (i.e., the proportion of real attendees among lowrisk patients) and accuracy (i.e., the proportion of appointments predicted correctly) were also estimated. The model performance in predicting non-attendance was prospectively validated using the same definitions of performance as for the retrospective validation. The only exception was considering balanced accuracy instead of raw accuracy because of class imbalance in the prospective validation.

\section{Pilot study}

The pilot study included all consecutive patients with at least one appointment scheduled between February 25 and
April 19, 2019, in either of the two involved services. The primary endpoint of the pilot study was the reduction of the non-attendance rate among patients at high risk of nonattendance (i.e., attendance probability below $50 \%$ according to the predictive model obtained). The week before the appointment, patients who were considered at high risk of non-attendance were randomly assigned to either a control or intervention group, balanced regarding age and gender. Right after randomization (i.e., one week before the appointment), patients allocated in the intervention group received a reminder phone call (up to three contact attempts) in which they were encouraged to either attend or early cancel the visit, whereas those in the control group did not receive any reminder. The outcomes related to the appointment reminder (i.e., whether the patient was reached, appointment cancellation or rescheduling, appointment attendance) were recorded.

\section{E. Statistical analysis}

Quantitative variables were presented as the mean and standard deviation (SD), and qualitative variables as frequency and percentage. Non-attendance rates were calculated by dividing the number of non-attended visits by the number of scheduled visits on a given period. Data from remote appointments, and negative days of waiting time (i.e., introduced in the program after the visit) were excluded from the analysis. Sensitivity, specificity, and accuracy were estimated directly from the contingency table of predicted and real missed appointments, whereas balanced accuracy was calculated as (sensitivity+specificity)/2. Qualitative variables were compared using the Chi-Square test, whereas quantitative variables were compared using analysis of variance (ANOVA). Correlations between quantitative variables were analyzed using the Pearson correlation test, whereas correlations between qualitative variables were analyzed with Cramer's V coefficient. The significance threshold was set at a bilateral alpha value of 0.05. All analyses were performed using the $\mathrm{R}$ software (version 3.6.1).

\section{SUPPLEMENTARY MATERIALS}

Supplementary file 1 contains the associations between variables and non-attendance for dermatology and pneumology services in Table S1 and Table S2, respectively. Correlation matrix among variables influencing nonattendance is presented in Table S3.

\section{ACKNOWLEDGMENT}

The authors would like to thank i2e3 Biomedical Research Institute for providing medical writing support.

\section{REFERENCES}

[1] L. R. LaGanga and S. R. Lawrence, "Clinic Overbooking to Improve Patient Access and Increase Provider Productivity*," Decis. Sci., vol. 38, no. 2, pp. 251-276, May 2007, doi: https://doi.org/10.1111/j.1540-5915.2007.00158.x.

[2] M. Bech, "The economics of non-attendance and the expected effect of charging a fine on non-attendees," Health Policy (New. 
York)., vol. 74, no. 2, pp. 181-191, 2005.

L. F. Dantas, J. L. Fleck, F. L. Cyrino Oliveira, and S. Hamacher, "No-shows in appointment scheduling - a systematic literature review," Health Policy (New. York)., vol. 122, no. 4, pp. 412-421, Apr. 2018, doi: 10.1016/j.healthpol.2018.02.002.

[4] M. Samorani and L. R. LaGanga, "Outpatient appointment scheduling given individual day-dependent no-show predictions," Eur. J. Oper. Res., vol. 240, no. 1, pp. 245-257, Jan. 2015, doi: 10.1016/J.EJOR.2014.06.034.

[5] R. Kopach et al., "Effects of clinical characteristics on successful open access scheduling," Health Care Manag. Sci., vol. 10, no. 2, pp. 111-124, May 2007, doi: 10.1007/s10729-007-9008-9.

[6] K. J. Hardy, S. V O’Brien, and N. J. Furlong, "Quality improvement report: Information given to patients before appointments and its effect on non-attendance rate," $B M J$, vol. 323, no. 7324, pp. 1298-1300, Dec. 2001, doi: 10.1136/bmj.323.7324.1298.

[7] R. Guy, J. Hocking, H. Wand, S. Stott, H. Ali, and J. Kaldor, "How effective are short message service reminders at increasing clinic attendance? A meta-analysis and systematic review.," Health Serv. Res., vol. 47, no. 2, pp. 614-32, Apr. 2012, doi: 10.1111/j.1475-6773.2011.01342.x.

[8] A. R. Pollastri, M. L. Pokrywa, S. J. Walsh, H. R. Kranzler, and J. Gelernter, "Incentive program decreases no-shows in nontreatment substance abuse research.," Exp. Clin. Psychopharmacol., vol. 13, no. 4, pp. 376-80, Nov. 2005, doi: 10.1037/1064-1297.13.4.376.

[9] Z. Chen, L. Fang, L. Chen, and H. Dai, "Comparison of an SMS text messaging and phone reminder to improve attendance at a health promotion center: A randomized controlled trial," $J$. Zhejiang Univ. Sci. B, vol. 9, no. 1, pp. 34-38, Jan. 2008, doi: 10.1631/jzus.B071464.

[10] I. Gurol-Urganci, T. de Jongh, V. Vodopivec-Jamsek, R. Atun, and J. Car, "Mobile phone messaging reminders for attendance at healthcare appointments," Cochrane Database Syst. Rev., vol. 2017, no. 12, Dec. 2013, doi: 10.1002/14651858.CD007458.pub3.

[11] A. Parikh, K. Gupta, A. C. Wilson, K. Fields, N. M. Cosgrove, and J. B. Kostis, "The Effectiveness of Outpatient Appointment Reminder Systems in Reducing No-Show Rates," Am. J. Med., vol. 123 , no. 6, pp. 542-548, Jun. 2010, doi: 10.1016/j.amjmed.2009.11.022.

[12] J. B. Norris, C. Kumar, S. Chand, H. Moskowitz, S. A. Shade, and D. R. Willis, "An empirical investigation into factors affecting patient cancellations and no-shows at outpatient clinics," Decis. Support Syst., vol. 57, pp. 428-443, Jan. 2014, doi: 10.1016/J.DSS.2012.10.048.

[13] O. Torres, M. B. Rothberg, J. Garb, O. Ogunneye, J. Onyema, and T. Higgins, "Risk factor model to predict a missed clinic appointment in an urban, academic, and underserved setting.," Popul. Health Manag., vol. 18, no. 2, pp. 131-6, Apr. 2015, doi: 10.1089/pop.2014.0047.

[14] D. Carreras-García, D. Delgado-Gómez, F. Llorente-Fernández, and A. Arribas-Gil, "Patient no-show prediction: A systematic literature review," Entropy, vol. 22, no. 6, Jun. 2020, doi: 10.3390/E22060675

[15] T. Therneau, B. Atkinson, and B. Ripley, "rpart: Recursive partitioning for classification, regression and survival trees.," CRAN R package version 4.1-15. 2019.

[16] L. Breiman, J. Friedman, C. Stone, and R. Olshen, "Classification and Regression Trees (Wadsworth Statistics/Probability)," New York CRC Press, 1984.

[17] M. U. Ahmad, A. Zhang, and R. Mhaskar, "A predictive model for decreasing clinical no-show rates in a primary care setting," Int. J. Healthc. Manag., 2019, doi: 10.1080/20479700.2019.1698864.

[18] E. S. Gromisch, A. P. Turner, S. L. Leipertz, J. Beauvais, and J. $\mathrm{K}$. Haselkorn, "Who is not coming to clinic? A predictive model of excessive missed appointments in persons with multiple sclerosis," Mult. Scler. Relat. Disord., vol. 38, Feb. 2020, doi: 10.1016/j.msard.2019.101513.

[19] J. Daggy et al., "Using no-show modeling to improve clinic performance," Health Informatics J., vol. 16, no. 4, pp. 246-259, Dec. 2010, doi: 10.1177/1460458210380521.

[20] X. Ding et al., "Designing risk prediction models for ambulatory no-shows across different specialties and clinics," J. Am. Med. Informatics Assoc., vol. 25, no. 8, pp. 924-930, Aug. 2018, doi: 10.1093/jamia/ocy002.

[21] C. Elvira, A. Ochoa, J. C. Gonzalvez, and F. Mochon, "MachineLearning-Based No Show Prediction in Outpatient Visits," Int. J. Interact. Multimed. Artif. Intell., vol. 4, no. 7, p. 29, 2018, doi: 10.9781/ijimai.2017.03.004.

[22] S. L. Chua and W. L. Chow, "Development of predictive scoring model for risk stratification of no-show at a public hospital specialist outpatient clinic," Proc. Singapore Healthc., vol. 28, no. 2, pp. 96-104, Jun. 2019, doi: 10.1177/2010105818793155.

[23] M.-Y. Suk, B. Kim, S. G. Lee, C. H. You, and T. H. Kim, "Evaluation of Patient No-Shows in a Tertiary Hospital: Focusing on Modes of Appointment-Making and Type of Appointment," Int. J. Environ. Res. Public Health, vol. 18, no. 6, p. 3288, Mar. 2021, doi: 10.3390/ijerph18063288.

[24] L. R. Chong, K. T. Tsai, L. L. Lee, S. G. Foo, and P. C. Chang, "Artificial Intelligence Predictive Analytics in the Management of Outpatient MRI Appointment No-Shows," Am. J. Roentgenol., pp. 1-8, Sep. 2020, doi: 10.2214/ajr.19.22594.

[25] R. L. Lagman et al., "'If You Call Them, They Will Come': A Telephone Call Reminder to Decrease the No-Show Rate in an Outpatient Palliative Medicine Clinic," Am. J. Hosp. Palliat. Med., 2020, doi: 10.1177/1049909120952322.

[26] K. C. Leong et al., "The use of text messaging to improve attendance in primary care: A randomized controlled trial," Fam. Pract., vol. 23, no. 6, pp. 699-705, Dec. 2006, doi: $10.1093 /$ fampra/cml044.

[27] M. A. G. Gomes, M. H. N. G. Abreu, F. M. Ferreira, F. C. Fraiz, and J. V. N. B. Menezes, "No-shows at public secondary dental care for pediatric patients: a cross-sectional study in a large Brazilian city," Cien. Saude Colet., vol. 24, no. 5, pp. 1915-1923, May 2019, doi: 10.1590/1413-81232018245.19312017.

[28] A. J. Miller, E. Chae, E. Peterson, and A. B. Ko, "Predictors of repeated 'no-showing' to clinic appointments," Am. J. Otolaryngol. - Head Neck Med. Surg., vol. 36, no. 3, pp. 411-414, May 2015, doi: 10.1016/j.amjoto.2015.01.017.

[29] H. Jensen, H. Møller, and P. Vedsted, "Characteristics of customary non-attenders in general practice who are diagnosed with cancer: A cross-sectional study in Denmark," Eur. J. Cancer Care (Engl)., vol. 28, no. 6, Nov. 2019, doi: 10.1111/ecc.13143.

[30] D. L. Wolff et al., "Rate and predictors for non-attendance of patients undergoing hospital outpatient treatment for chronic diseases: A register-based cohort study," BMC Health Serv. Res., vol. 19, no. 1, p. 386, Jun. 2019, doi: 10.1186/s12913-019-42089 .

[31] M. M. Coleman, L. N. Medford-Davis, O. H. Atassi, A. SilerFisher, and C. A. Reitman, "Injury type and emergency department management of orthopaedic patients influences follow-up rates," J. Bone Jt. Surg. - Am. Vol., vol. 96, no. 19, pp. 1650-1658, Oct. 2014, doi: 10.2106/JBJS.M.01481. 